\title{
Physics Performance of the Barrel RPC System of the HARP Experiment
}

\author{
M. Bogomilov, A. Artamonov, S. Giani, D. Kolev, J. Panman, R. Tsenov, and I. Tsukerman
}

\begin{abstract}
The physics performance of the barrel RPC system of the HARP experiment is described. In the barrel two sets of fifteen identical resistive plate chambers (RPCs) have been operated in 2001 and 2002 as a part of the HARP experiment at the CERN $P S$ accelerator. For the first time under real experimental conditions RPCs have bean applied for particle identification (PID) by measuring the particle's time-of-flight (ToF). The procedure developed for the RPC calibration, based on reconstructed tracks in the HARP Time Projection Chamber (TPC), is described in detail. Intrinsic RPC time resolutions of 141 ps and a combined time resolution of the large angle TOF system of $180 \mathrm{ps}$ are obtained. The effective resolution of the comparison of predicted and measured ToF is 305 ps in the region of interest for this experiment. The PID capabilities of the system are demonstrated. An average efficiency of the RPC counters of about $97 \%$ is measured.
\end{abstract}

Index Terms-Gaseous detector, HARP, particle identification, resistive plate chamber (RPC), time-of-flight (TOF).

\section{INTRODUCTION}

$\mathbf{T}$ HE HARP experiment [1], [2] has been designed to measure hadron production cross-sections on fixed targets with a precision of a few percent over almost the full solid angle. A set of solid and liquid targets spanning a large range in atomic number was exposed to beams of protons and pions with momenta between $1.5 \mathrm{GeV} / \mathrm{c}$ and $15 \mathrm{GeV} / \mathrm{c}$. The elements used ranged from hydrogen to lead. HARP took 450 million physics triggers, collected data for about 300 different settings and recorded $30 \mathrm{~TB}$ of information from August 2001 to October 2002.

The setup of the HARP experiment is shown in Fig. 1. A detailed description of the detector and its performance are given in [1], [2]. The spectrometer can be subdivided into three main systems:

- Beam and trigger detectors provide tracking and identification of beam particles, and trigger decisions.

- Forward detectors provide tracking, momentum measurement and identification of secondary particles at angles less than $20^{\circ}$ with respect to the beam axis.

Manuscript received September 5, 2006; revised December 4, 2006. This work was supported in part by the Swiss National Science Foundation and the Swiss Agency for Development and Cooperation in the framework of the program SCOPES - Scientific co-operation between Eastern Europe and Switzerland.

M. Bogomilov, D. Kolev, and R. Tsenov are with the Department of Atomic Physics, Faculty of Physics, St. Kliment Ohridski University of Sofia, BG-1164 Sofia, Bulgaria.

A. Artamonov and I. Tsukerman are with the Institute for Theoretical and Experimental Physics, 117218 Moscow, Russia.

S. Giani and J. Panman are with the European Organization for Nuclear Research (CERN), CH-1211 Geneva 23, Switzerland.

Digital Object Identifier 10.1109/TNS.2006.890323

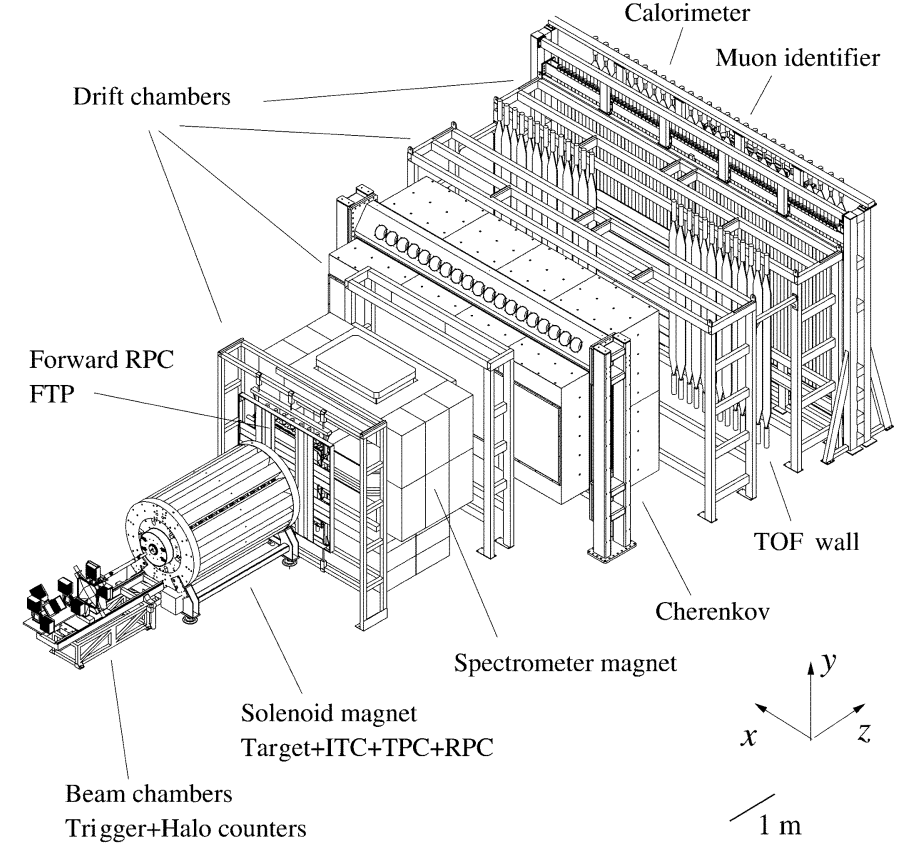

Fig. 1. General layout of HARP spectrometer.

- Large angle detectors deal with tracking, momentum measurement and particle identification at large production angles.

The large angle detector system consists of:

- Time Projection Chamber (TPC). This has a cylindrical form with a length of $150 \mathrm{~cm}$ and a diameter of $80 \mathrm{~cm}$. It is located inside a solenoid magnet producing a $0.7 \mathrm{~T}$ field. Charged secondary particles ionize the gas along their (curved) trajectories in the TPC. The produced electrons and ions drift to the electrodes owing to the electric field applied parallel to the TPC axis of symmetry. Thus, the TPC measures momentum, trajectory and ionization energy losses of particles emitted from the target at large angles $\left(20^{\circ}<\theta<160^{\circ}\right)$ with respect to the incoming beam.

- Resistive Plate Chambers are arranged in the shape of a barrel around the outer field cage of the TPC. A more detailed description of this system is given in the following sections.

- Target station and Inner Trigger Cylinder (ITC) are situated inside TPC volume, in a truncated inner field cage.

This paper concentrates on the performance of the detector system obtained for physics parameters such as time resolution and efficiency and the calibration procedures developed to achieve this performance. Section II contains a brief description 


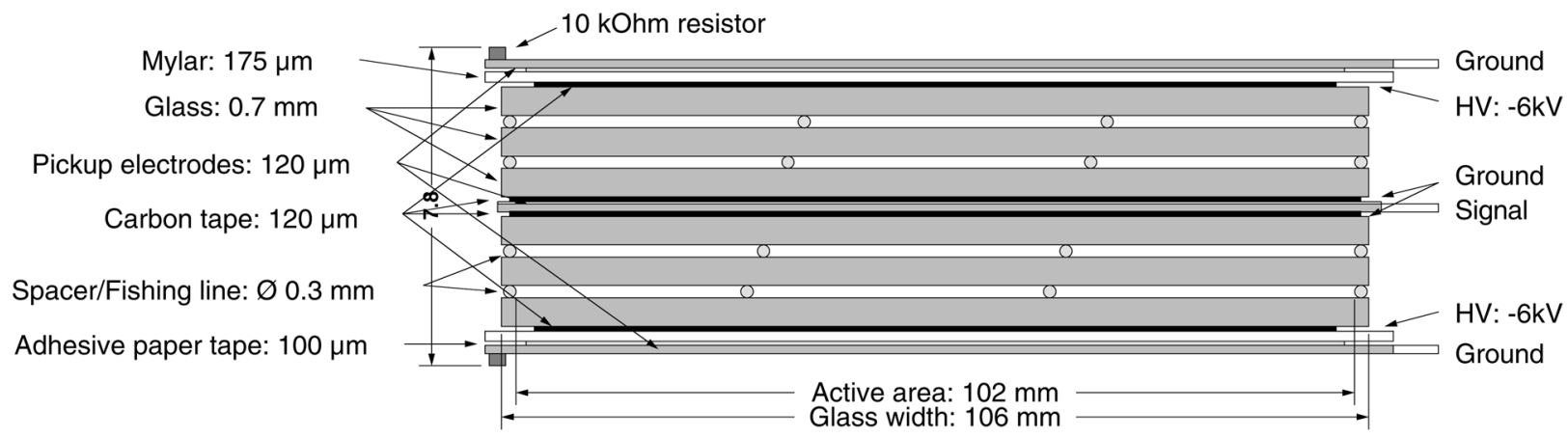

Fig. 2. Cross-section of RPC glass stack. Six glass plates form four $0.3 \mathrm{~mm}$ gaps using fishing lines as spacer. The only signal electrode is situated in the middle of the stack.

of the RPC detector layout to serve as a basis for the subsequent sections. For a more complete description of the hardware see [2] and [3]. The calibration procedures necessary to achieve the performance of the chambers are described in Section III. The system performance in terms of physics parameters is given in Section IV.

\section{HARP RPC SYSTEM}

In the HARP experiment, particles produced at large angles with respect to the incoming particle beam are detected by the Time Projection Chamber - the main tracking and particle identification device in that region. In the momentum range 125 $\mathrm{MeV} / \mathrm{c}-250 \mathrm{MeV} / \mathrm{c}$ ionization energy losses $d E / d x$ of electrons and pions are similar and they cannot be separated using $d E / d x$ alone. On the other hand, the background from $\pi^{0}$ decay photons converting to $\mathrm{e}^{ \pm}$pairs which mimic charged pion production is expected to be significant in this momentum region. A system of resistive plate chambers is used as a complementary PID device for particles emerging with large production angles. Owing to the relatively low momenta of the secondary particles emitted at large angles, PID can be performed by ToF measurements. A time resolution of $\sim 200 \mathrm{ps}$ is required to separate pions and electrons in the above momentum range when they traverse the TPC over the shortest distance $(\sim 400 \mathrm{~mm})$. ToF measurements with such a resolution also help in pion-proton separation up to $\sim 1 \mathrm{GeV} / \mathrm{c}$.

\section{A. Chamber Design}

The HARP RPCs design is based on a prototype developed for the ALICE experiment [4].

The RPCs are constructed as a four-gap stack of glass plates. The gap size is precisely set to $0.3 \mathrm{~mm}$ by interposing a fishing line (nylon mono-filament) with suitable diameter between the plates. The stack consists of two identical structures of three glass plates each, arranged symmetrically on both sides of a central readout electrode. The glass plates are $0.7 \mathrm{~mm}$ thick and made of standard float glass with a specific resistivity of $\sim 10^{13} \Omega \mathrm{cm}$. A view of the cross-section through the short side of the glass stack is shown in Fig. 2. The negative high voltage is applied to the glass plates by means of a coated graphite with resistivity of $200 \mathrm{k} \Omega / \square$ on the two outer glass plates of both sets. A chamber is $1920 \mathrm{~mm}$ long, $104 \mathrm{~mm}$ wide, and $7.8 \mathrm{~mm}$ thick. Each chamber is housed in an aluminum box with dimensions $2 \mathrm{~m} \times 150 \mathrm{~mm} \times 10 \mathrm{~mm}$.

A single readout electrode, located in the center of the glass stack, collects signals from all four gaps. It is segmented into 64 rectangular strips. Eight strips are connected to one pre-amplifier forming a readout channel. Thus, each chamber has eight readout channels, in the following referred to as pads, and numbered from 1 to 8 starting from the most upstream one.

\section{B. Detector Layout}

The RPC system comprises 46 identical chambers. Sixteen RPCs are installed downstream of the TPC, perpendicular to the beam at about $2 \mathrm{~m}$ distance from the target. The remaining thirty RPCs are arranged around the TPC in two staggered layers forming a barrel. They cover polar angles from $17^{\circ}$ to $142^{\circ}$ with respect to the beam axis and $2 \pi$ in azimuthal angle with a small $13 \mathrm{~mm}$ overlap between the layers. The set of two layers fits into a $\sim 25 \mathrm{~mm}$ radial space between the TPC and the coils of the solenoid magnet as shown in Fig. 3. The readout electrodes of the inner and outer layer are located at radial distances of $R_{\text {in }}=421 \mathrm{~mm}$ and $R_{\text {out }}=434.5 \mathrm{~mm}$, respectively.

The front-end electronics of each channel consists of a preamplifier board mounted on the chamber and a combined discriminator/splitter module.

Pre-amplifiers are based on AD8009 chip operating with an amplification factor of $\sim 30$. The amplified signals are transmitted through mini coaxial cables over a distance of $0.8 \mathrm{~m}-2.5 \mathrm{~m}$ (depending on the channel in question) to a passive connector board and from there over a distance of $5 \mathrm{~m}$ through Lemo $50 \Omega$ cables to a custom-made leading edge discriminator and splitter module. The discriminator threshold is put at $5 \mathrm{mV}$. Each signal is amplified once more in the discriminator/splitter module (with a factor $\approx 3$ using the same AD8009 chip) and split into two separate signals. One of the signals is discriminated and sent via $80 \mathrm{~m}$ twisted pair cable to a Time-to-Digital Converter (TDC), model CAEN V775. The other signal is sent through another $80 \mathrm{~m}$ twisted pair cable to a Charge-To-Digital Converter (QDC), model CAEN V792. The set up of the readout chain is given in Fig. 4.

\section{Operational Parameters}

The RPCs were operated in avalanche mode at a voltage of $-6 \mathrm{kV}$ between outer and central electrodes and with a gas 

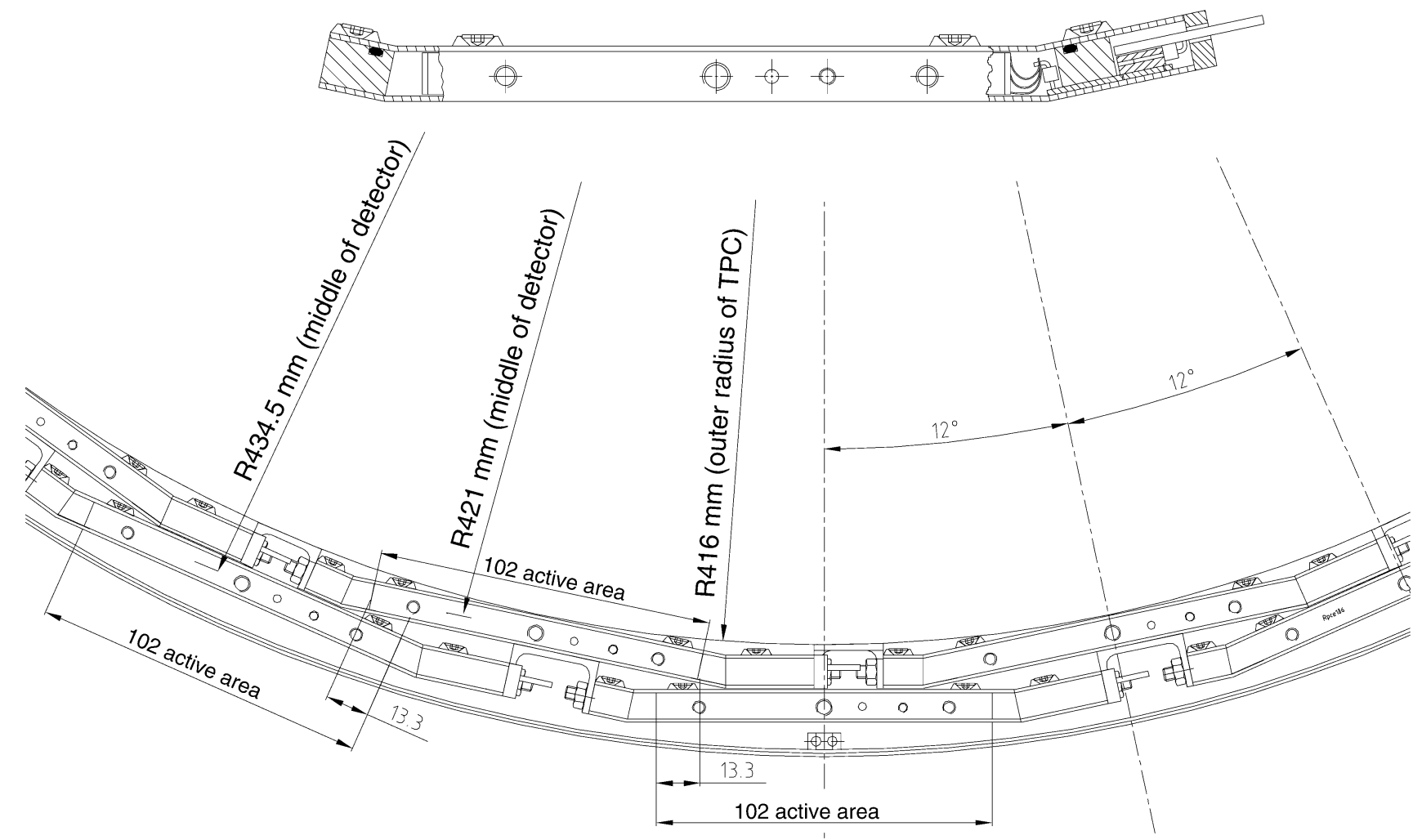

Fig. 3. Schematic layout of the barrel RPC in the $x-y$ plane (perpendicular to the beam). Thirty RPCs are arranged in two staggered layers covering the full azimuthal angle including some overlap.
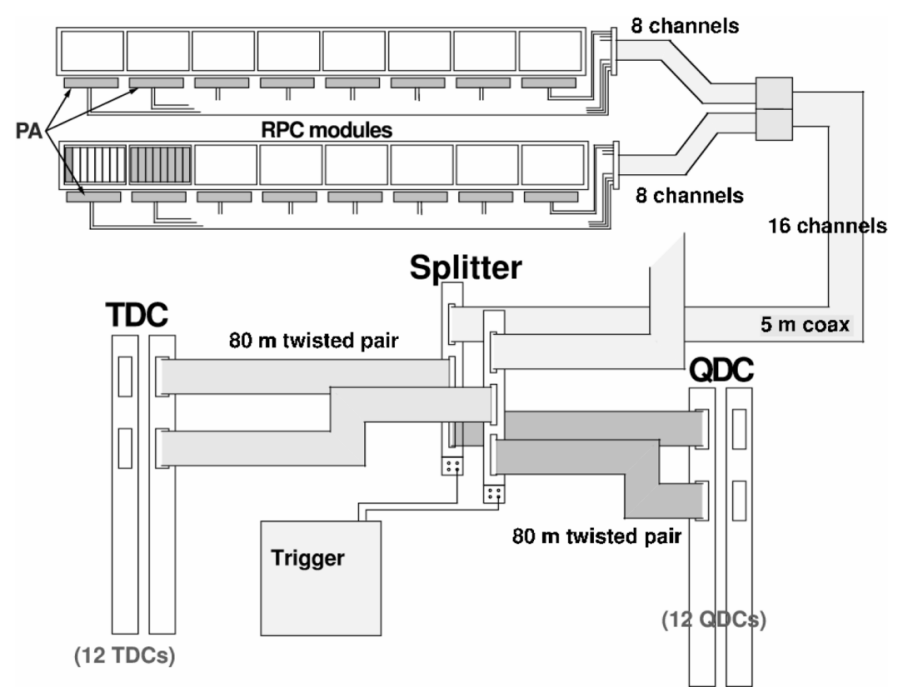

Fig. 4. Layout of the readout chain. The signals coming from two chambers are sent through mini-coax cables to a passive connector and then to a splitter module. One part of the signal is amplified, discriminated and sent to TDC for time digitization, the second one is sent directly to QDC for amplitude digitization.

mixture of $90 \% \mathrm{C}_{2} \mathrm{~F}_{4} \mathrm{H}_{2}, 5 \% \mathrm{SF}_{6}$, and $5 \% \mathrm{C}_{4} \mathrm{H}_{10}$ at a gas flow of $\sim 1$ volume change per hour. Two neighboring RPCs were supplied through one gas line. During the 2002 data taking period only three channels in the barrel (out of 240) lost their signals which is considered as an acceptable level of reliability.
The ambient temperature fluctuated in the range of $27^{\circ} \mathrm{C}-$ $30^{\circ} \mathrm{C}$ for the barrel and $20^{\circ} \mathrm{C}-35^{\circ} \mathrm{C}$ for the forward chambers (varying with the hall temperature).

Typical random noise rates were in the range of $200 \mathrm{~Hz}-$ $300 \mathrm{~Hz}$ per chamber, i.e., $\leq 1 \mathrm{kHz} / \mathrm{m}^{2}$, which is an acceptable level compared to the typical particle rate $10 \mathrm{kHz} / \mathrm{m}^{2}$ over the area covered by the barrel RPC. Significantly higher noise rates were observed in only a few chambers. In those chambers the origin of this was traced back to mechanical problems with the glass stack.

More details of the design, layout and operational parameters of the HARP RPCs can be found in [3].

\section{Calibration Procedure}

A precise calibration of the RPC sub-detector is needed before it can be used for time-of-flight measurements. The aim of the calibration is to develop a procedure that transforms data read out from a TDC channel to a flight-time for particles from the production point in the target to the RPC pad feeding that particular TDC channel. The calibration procedure described below concerns only barrel RPCs. It is developed by using reconstructed charged particle tracks in the TPC from interactions produced by beams of positive particles with momenta of $3 \mathrm{GeV} / \mathrm{c}, 5 \mathrm{GeV} / \mathrm{c}$, and $8 \mathrm{GeV} / \mathrm{c}$ bombarding $0.05 \lambda_{\text {int }}$ thick Ta, $\mathrm{Pb}, \mathrm{Sn}$, and $\mathrm{Cu}$ targets.

\section{A. Basic Selection Criteria}

1) The selection of samples of pions and protons is done by exploiting the average $d E / d x$ measured in the TPC along 


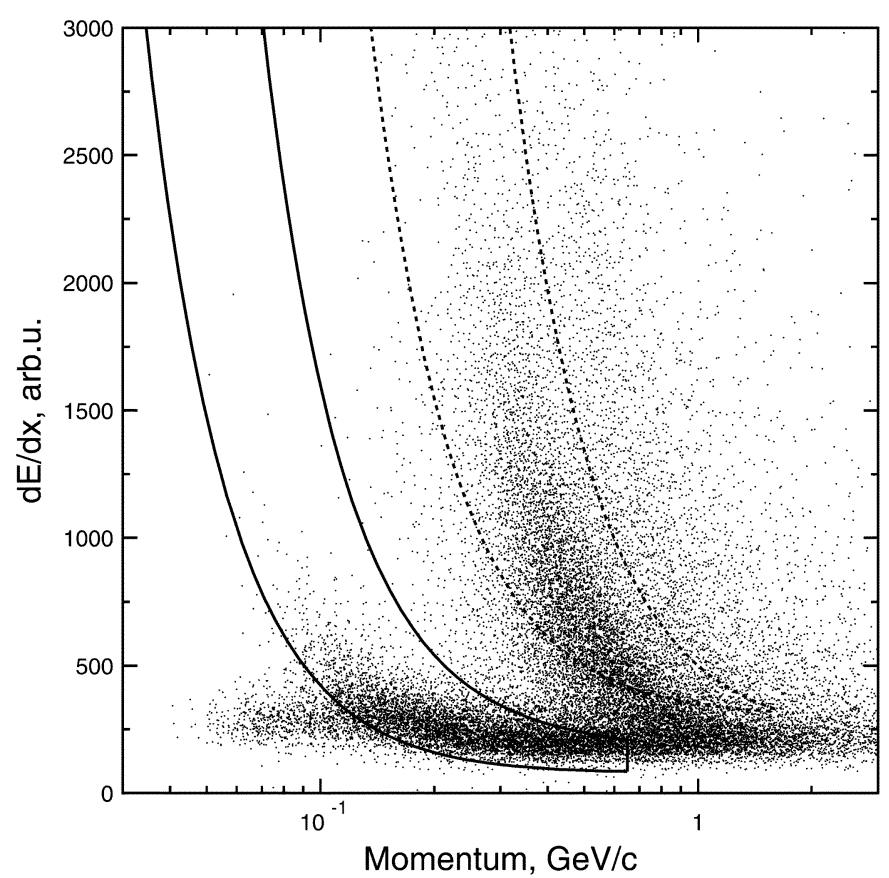

Fig. 5. Cuts for selection of $\pi^{ \pm}$and protons in the "energy loss-momentum" plot. A pure pion sample is obtained selecting tracks falling between the two solid lines (momentum $<0.65 \mathrm{GeV} / \mathrm{c}$ ). Protons are selected between the two dotted lines.

their trajectory. The $d E / d x$ of selected tracks of protons and pions is enclosed within the cuts drawn in Fig. 5.

2) The selection of the particle charge is obtained from the orientation of the track curvature measured by the TPC.

3) The following cuts on the TPC track quality are applied: the number of points on the track is required to be at least ten and tracks are required to originate from the target.

4) Hits in RPC channels with measured signal charge less than 300 and more than 3840 QDC units are rejected. This protects against a badly defined behavior of the time-charge correction for small charges (see Fig. 6) and charge overflow without incurring a significant reduction of the statistics.

\section{B. Conversion of TDC Measurements to Particle ToF}

The conversion of the measurements of signal time and pulseheight in the TDCs and QDCs of the RPC readout is achieved via the following steps:

1) The TDC channel scale is converted to physical time using the measured characteristics of every TDC channel. The nominal width of a TDC channel increment is $35 \mathrm{ps}$. This value is only approximate. The width of each TDC channel count has been measured and used for the above conversion. The average value of all TDC channels is $36.5 \mathrm{ps}$.

2) The arrival time of the beam particle at the target is subtracted. The Beam ToF system [5] provides time measurements for beam particles and gives the arrival time of the beam particle at the target $(z=0)$ in the common time reference frame of the detector.

3) Corrections for the transit time of the signal within the pad and from the pad to the pre-amplifier are applied. These

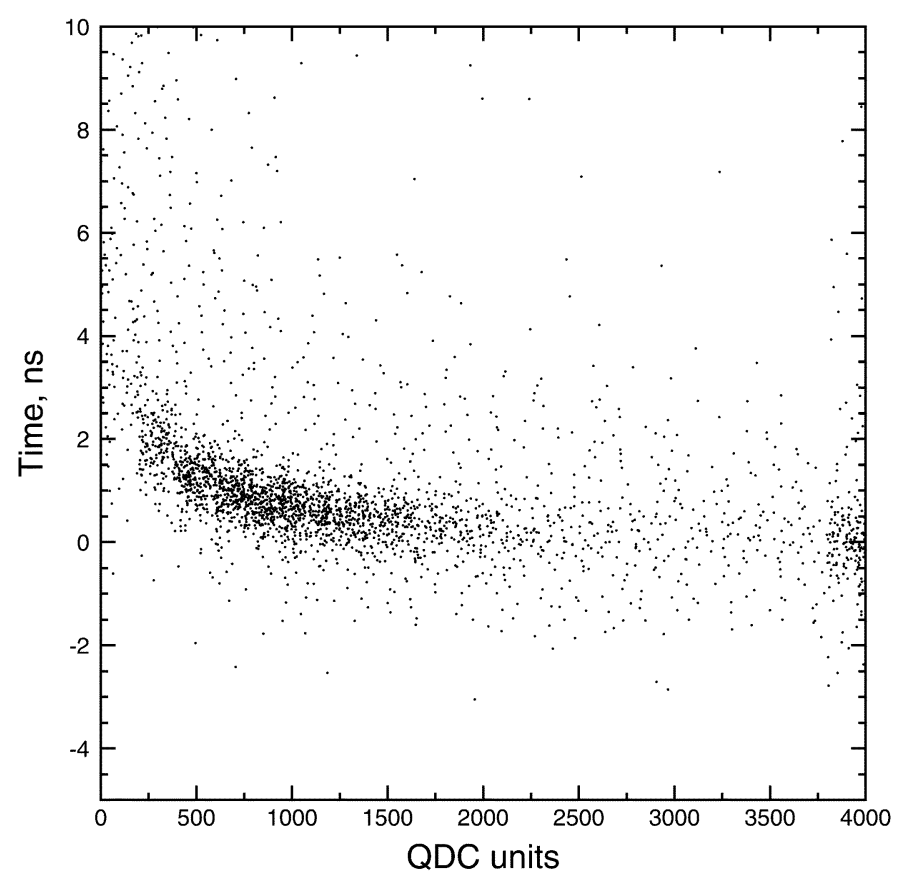

Fig. 6. Time-charge dependence for negative pions. The recorded TDC time is larger for smaller QDC charges because of the slower rise of the signal. Pions predominantly create charges less than 1500 QDC units.

corrections have been determined in special test beam measurements described in [6].

4) A run-by-run time offset is applied (see Section III.D).

\section{Time-Charge Correction}

A specialized discriminator with fixed level of discrimination is used to reshape the signals before they are input to the TDC. As a result the signals with different charges cross the discrimination level with different delays that results in so called time-walk. Hence a dependence between the charge measured by the QDC and recorded TDC counts is introduced. This relationship is referred to as time-charge dependence. A typical example is shown in Fig. 6.

An analytical approximation for the time-charge dependence has been derived from two-dimensional distributions like the one shown in Fig. 6. Similar distributions have been collected for each pad-ring. From these distributions the mean values of the difference of the recorded and predicted particle ToF time have been obtained in $\sim 200$ QDC units wide bins of measured charge. The variation of these mean values with the signal charge is well approximated with the function:

$$
t(q)=a_{0}+\frac{a_{1}}{q-q_{0}}+\frac{a_{2}}{\left(q-q_{0}\right)^{2}}+\frac{a_{3}}{\left(q-q_{0}\right)^{3}},
$$

where $t(q)$ is the time delay and $q$ is the measured charge.

It has been observed that the signals from $\pi^{-}$and $\pi^{+}$exhibit a very similar time-charge dependence (as expected), while signals induced by protons behave differently. Pions and protons have been identified by means of average $d E / d x$ of their tracks reconstructed in the TPC, see Fig. 5. The evaluated analytical approximations for pion and proton time-charge corrections are shown in Fig. 7. As can be seen, proton time delays are on average $\sim 400 \mathrm{ps}$ smaller than those of pions. 


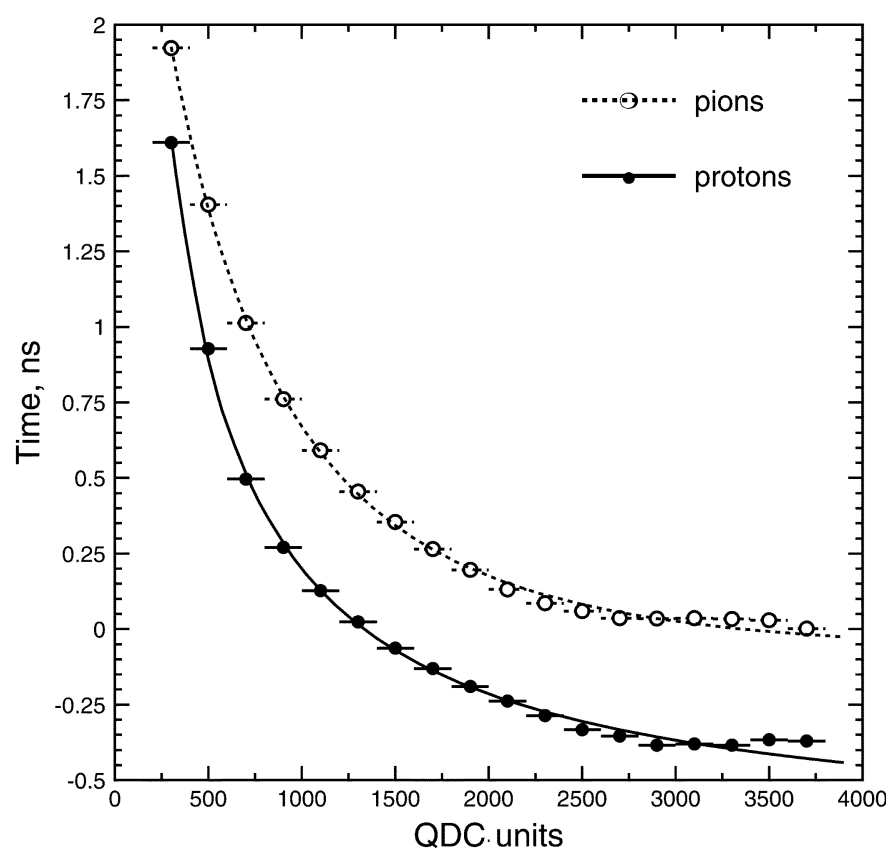

Fig. 7. Time-charge corrections for pions and protons, all pads combined.

The origin of this difference is not yet clear. It might be related to different energy losses of pions and protons in the RPC gas gaps and glass walls, hence a possibly different ionization and different gas amplification regime. Since the observed rise-time of the signals is several $\mathrm{ns}$, a small difference in signal shape between heavily ionizing particles and minimum ionizing particles could induce such an effect. In particular, effects below a ns are contained within the expected avalanche formation time. Exposing one or several RPCs directly to beams of pions and protons will give more insight into the origin of this behavior. However, this study is beyond the scope of the present article. Here, in order to test any dependence on the energy-loss of the particles in the gas gaps we have compared the time-charge dependence of protons above $1 \mathrm{GeV} / \mathrm{c}$ with relativistic pions and heavily ionizing protons. We observe that the time-charge dependence of the faster protons (with energy-losses close to those of the relativistic pions) is equal to that of pions.

This result suggests that a dependence on the primary ionization in the gas gaps plays a role. To investigate this hypothesis, the pulse-height spectra for pions and protons of different momenta were collected. As a measure of pulse-height the position of the peak (most probable charge) was determined. Fig. 8 displays the behavior of the peak position for pions (left panel) and protons (right panel). The dependences are shown for different pad-rings. Tracks crossing pad-ring 3 are nearly perpendicular to the RPC while the angle increases with pad-ring number, to about 30 degrees at ring number 7 . One observes a strong dependence on $d E / d x$ (as seen for the heavily ionizing protons in this momentum range) and an additional dependence on incident angle. Thus the pulse-height is not independent from the primary ionization, but depends both on ionization density and total primary ionization. Upon further inspection of the pulseheight one observes that the most probable value is not proportional to the total primary ionization calculated for the velocity of the particles. This observation suggests that the development of the avalanche can be faster for higher primary ionization,
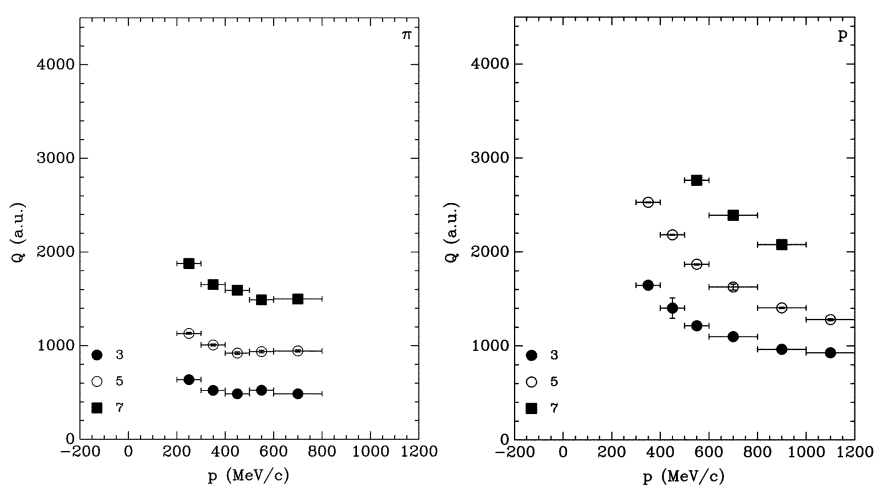

Fig. 8. Most probable charge $Q$ for pions (left panel) and protons (right panel), for pad-rings 3,5 , and 7 .
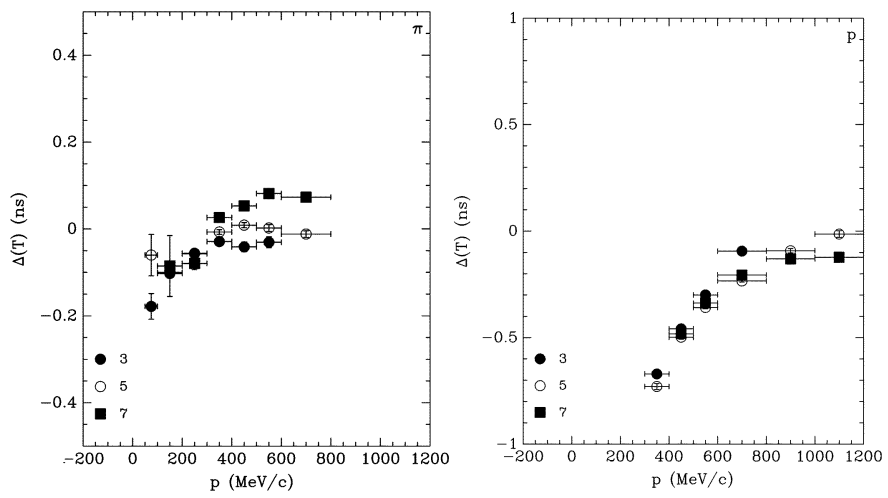

Fig. 9. Difference of the measured time offset from the expected time offset for pions (left panel) and protons (right panel), for pad-rings 3, 5, and 7.

whether due to higher ionization density or a larger track length in the gap, thus changing the shape of the pulse and not only the height. If this hypothesis is correct, the time difference between the observed signal (corrected using the overall time-charge relation per pad) and the expected arrival time calculated on the basis of the track length and particle momentum should show a dependence as a function of momentum for the same set of pad-rings. The behavior shown in Fig. 9 suggests a clear dependence on ionization density $(d E / d x)$ and a dependence on different path lengths at fixed $d E / d x$ only for low $d E / d x$. One also observes similar time delays for pions and protons at fixed particle velocity.

In the HARP experiment, the RPC system is intended mainly for separation of electrons and pions below $250 \mathrm{MeV} / \mathrm{c}$. Hence, the observed peculiarity of the sensitivity of the time-charge dependence on primary ionization is not important and the dependence measured with pions is used in the analysis below and in the standard HARP data processing.

\section{Temperature Correction}

During data analysis it was observed that TDC measurements depend on ambient temperature in the experimental hall. A temperature change of about 10 degrees causes a time drift of about $500 \mathrm{ps}$. In the temperature range of interest a linear dependence of the RPC time response on ambient temperature was found with a slope of $(49 \pm 5) \mathrm{ps} / \mathrm{deg}$ [7]. This temperature drift together with other smaller shifts (e.g., due to hardware replacements) of measured ToF were corrected as follows. For a given 


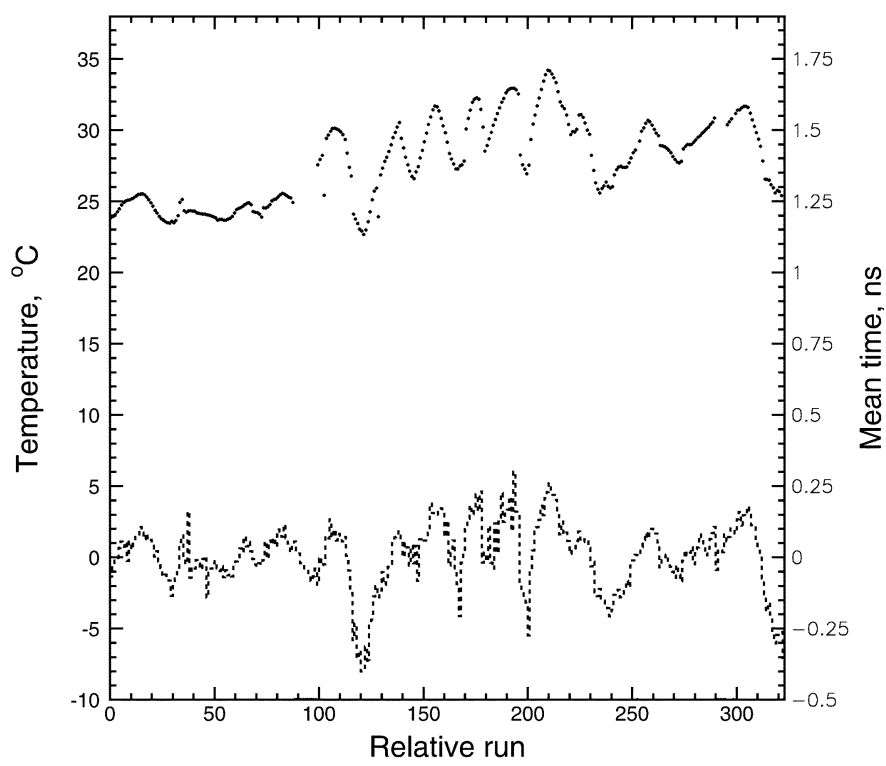

Fig. 10. Run temperature (upper curve, corresponding to left ordinate) and run average time-of-flight difference (bottom curve, corresponding to right ordinate) for the data used for RPC calibration. On the $x$-axis the run numbers are relative to an arbitrary offset.

set of measurements an integral average value of the ToF differences (which is measured ToF, see III.B, minus predicted ToF from track momentum and length) was computed. The average ToF difference for each run in that set was also calculated and compared to the integral average value. The difference between the two was used as specific run-by-run correction being more general than a correction for temperature variations alone. This correction improves the intrinsic RPC ToF resolution by about 30 ps.

The observed correlation between the average ToF difference per run and the ambient temperature during the data taking period of the present study is illustrated in Fig. 10. It should be noted that the chambers themselves are positioned inside the (water cooled) TPC magnet and therefore undergo only very small temperature variations. Hence the observed temperature dependence is most likely due to the ambient temperature variations to which the electronics (discriminators) are exposed.

\section{E. Calculation of Time Offsets $t 0$}

The time-offset constants $t 0$ account for propagation time of the RPC signals through cables and electronic modules of each channel (pad). The determination of these time offsets has been performed according to the following equation:

$$
t 0=t_{\text {RPC }}-t_{\text {charge }}-t_{\text {beam }}-t_{\text {temp }}-t_{\text {trans }}-t_{\text {pred }}^{\pi}
$$

where:

$-t_{\mathrm{RPC}}$ is measured by the TDC time, converted to ns,

$-t_{\text {charge }}$ is the time-charge correction,

- $t_{\text {beam }}$ is the arrival time of the beam particle at the target,

$-t_{\text {temp }}$ is run-by-run correction,

$-t_{\text {trans }}$ is the signal transition time within the pad and to the pad pre-amplifier,

$-t_{\mathrm{pred}}^{\pi}$ is the ToF for pions computed from track momentum and length. (a) channel No. 52

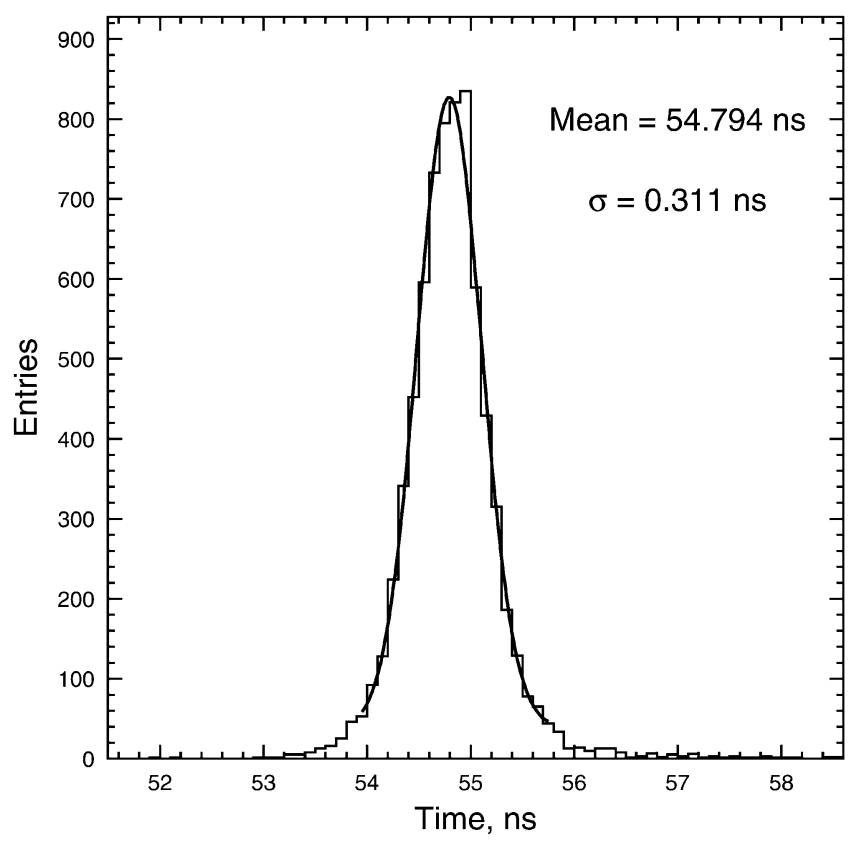

(b)

channel No. 238

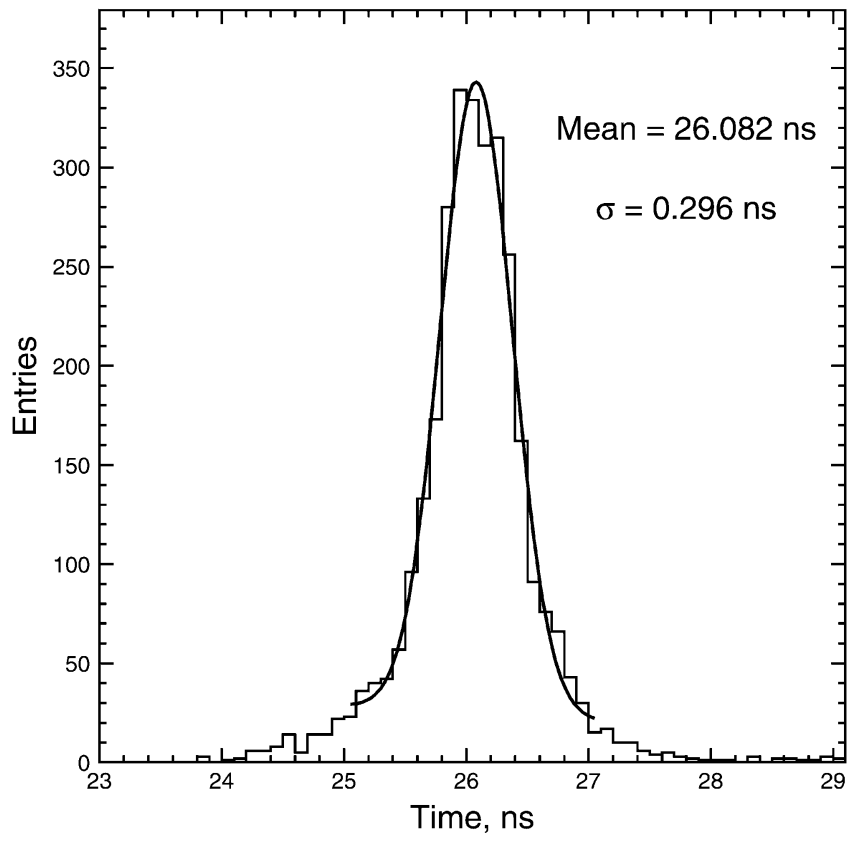

Fig. 11. Examples of $t 0$ distributions for two RPC pads. The $t 0$ for a given pad is the mean of the Gaussian fit. (a) $t 0=54.794 \mathrm{~ns}$ with $\sigma_{t 0}=0.311 \mathrm{~ns}$; (b) $t 0=26.082 \mathrm{~ns}$ with $\sigma_{t 0}=0.296 \mathrm{~ns}$.

The above computation is made for each track that has a signal in the pad. The $t 0$ for this pad (RPC channel) is obtained as the mean of the Gaussian fit to the distribution of $t 0$ over all hits. Examples are given in Fig. 11.

The calculation of a new set of $t 0$ constants is mandatory when electronic equipment has been changed and is recommended to be done for groups of data sets (setting) taken close in time (few days). Here setting means a certain combination of beam momentum, target and a given apparatus tuning. 
ring No. 6

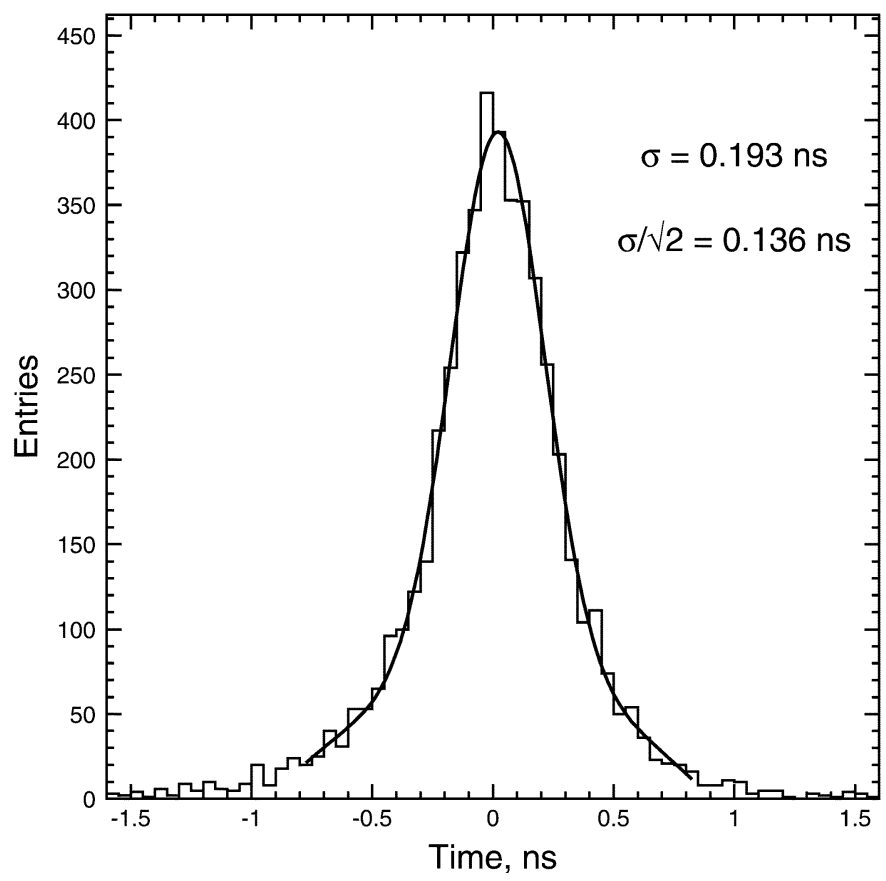

Fig. 12. Time difference for overlapping pads in pad-ring 6. The central part of the distribution is fitted by a Gaussian (solid curve) and a second order polynomial. The $\sigma$ of the Gaussian is $0.193 \mathrm{~ns}$ and $\sigma / \sqrt{(2)}=0.136 \mathrm{~ns}$.

An estimate of the stability of the RPC calibration can be obtained by taking differences of $t 0$ constants for different groups of settings. The results show that the distributions of differences have a Gaussian shape with $\sigma \approx 35$ ps.

\section{RPC PERFORMANCE}

The physics performance of the barrel RPC system has been studied using the same selection criteria as described in Section III.A. The time resolution is estimated using full available statistics and selecting pions only.

\section{A. Intrinsic RPC Time Resolution}

The difference of the time measured by two overlapping pads when crossed by one and the same track can be used to estimate the intrinsic resolution of the RPC detectors. A typical distribution of this time difference is shown in Fig. 12. The $\sigma$ of the Gaussian fit of the central part divided by $\sqrt{2}$ represents the intrinsic RPC time resolution, $136 \mathrm{ps}$ in this case. Averaged over all barrel pad-rings the resolution is $141 \mathrm{ps}$. The observed variation of the time resolution with signal charge is presented in Fig. 13. An apparent deterioration of the resolution for larger signal charges could be attributed to a change from avalanche to streamer working mode of the RPC, when the crossing particle, by some interaction mechanism, creates a big charge in the chamber's gas gaps. Further investigation of the phenomenon calls for a detailed physical model of RPC signal formation and, as mentioned above, is beyond the scope of this article. Also a contribution of accompanying particles correlated with the particle measured in the TPC, such as $\delta$-electrons can be present. However, the effect is not crucial for the data analysis because

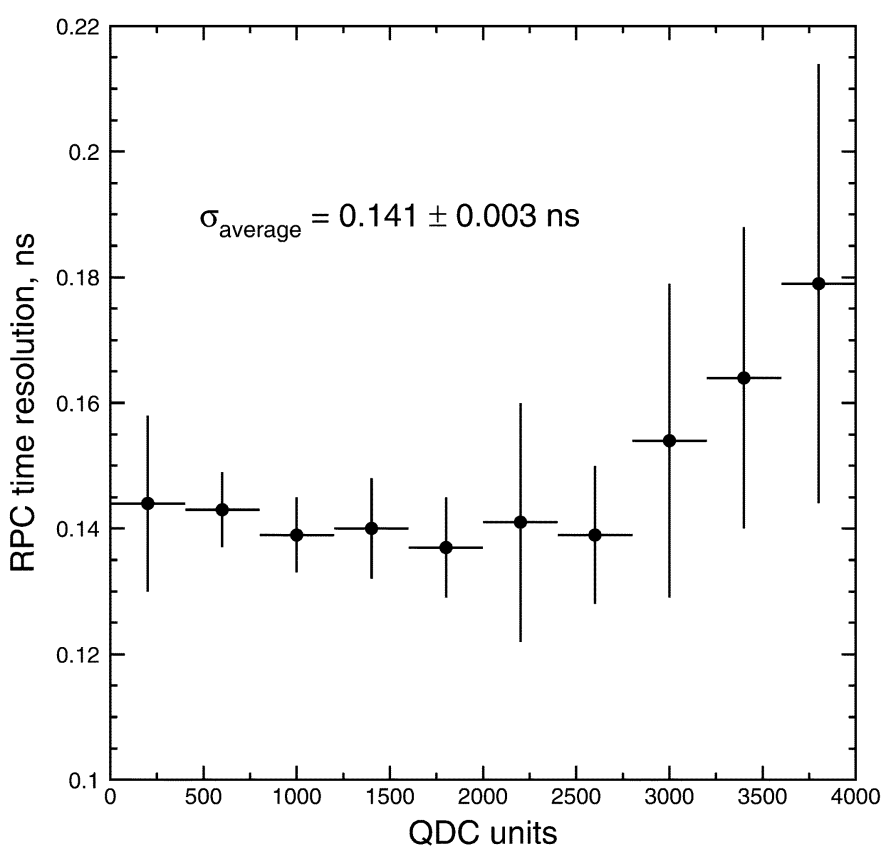

Fig. 13. Intrinsic RPC time resolution versus QDC charges, all rings are integrated. Plotted values are already divided by $\sqrt{2}$. The average value is $141 \mathrm{ps}$.

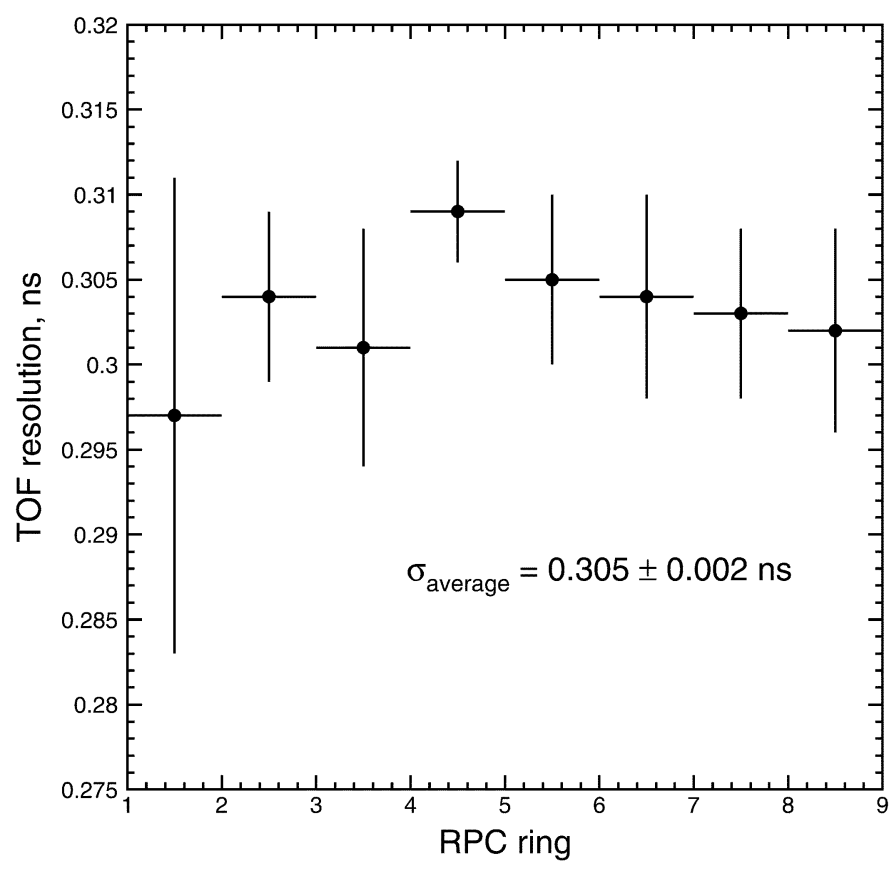

Fig. 14. $t_{\text {tof }}$ uncertainty measured for pions (the $\sigma$ of a Gaussian fit to the central part of distributions) versus RPC ring number for different QDC charge ranges. The distribution represents the combined time resolution of RPC, TPC, and Beam ToF detectors. The average value is $305 \mathrm{ps}$.

only few percent of the registered particles create charges bigger than 2500 QDC units.

\section{B. Overall Time Resolution of the Barrel ToF System}

The overall system ToF resolution accumulates, besides intrinsic RPC resolution, uncertainties in measurements delivered by two other HARP sub-detectors, namely the Beam ToF set 


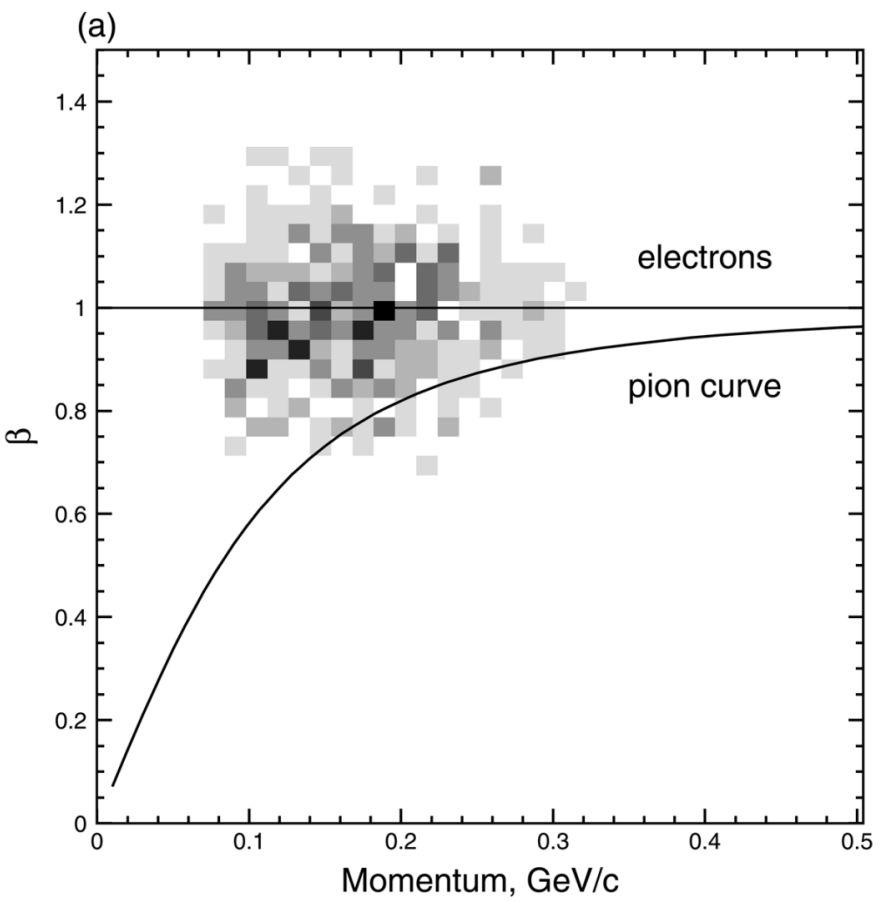

(b)

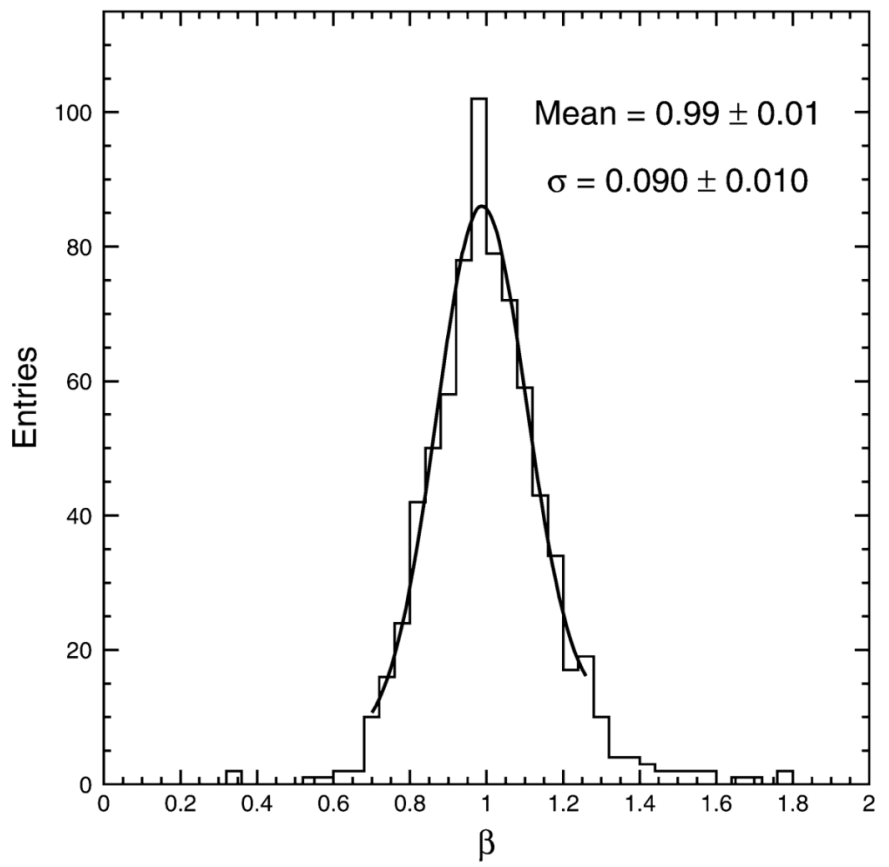

Fig. 15. A track sample enriched in $\mathrm{e}^{ \pm}$. Selected are pairs of "symmetric" tracks with opposite charge, close in "transverse momentum-production angle" space and with common origin. (a) relativistic velocity $\beta$ vs. track momentum. The solid lines represent the theoretical curves for electrons and pions. It is seen that the pion contamination in the sample is very small. (b) projection onto the $\beta$ axis. The standard deviation of the Gaussian fitted to the peak is $9.0 \%$.

and the TPC. Hence, the global uncertainty of the ToF $\sigma\left(t_{\text {tof }}\right)$ measured by the RPC can be expressed by:

$$
\sigma^{2}\left(t_{\mathrm{tof}}\right)=\sigma^{2}\left(t_{\mathrm{RPC}}\right)+\sigma^{2}\left(t_{\mathrm{BEAM}}\right)+\sigma^{2}\left(t_{\mathrm{TPC}}\right)
$$

where: $\sigma\left(t_{\mathrm{RPC}}\right)$ is the intrinsic RPC time resolution, estimated as $141 \mathrm{ps} ; \sigma\left(t_{\mathrm{BEAM}}\right)$ is the uncertainty of the arrival time of beam particle at the target, estimated to be $\sim 70$ ps [5]; and

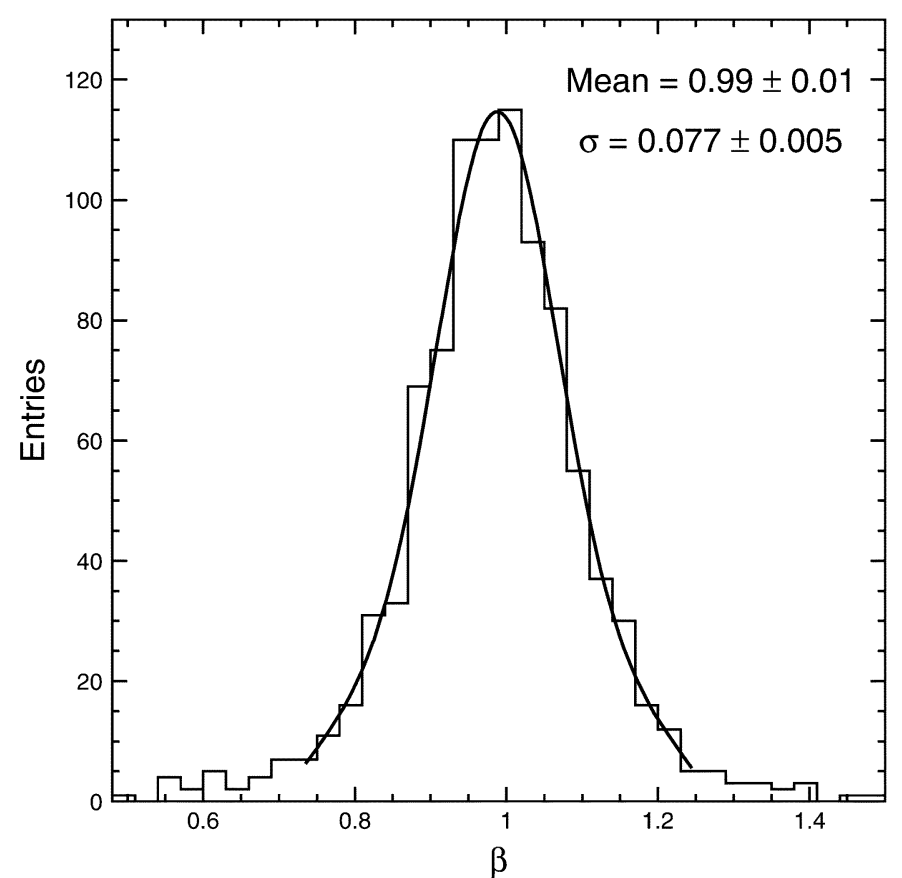

Fig. 16. $\mathrm{e}^{ \pm}$selected by a constraint on mean $d E / d x$ along the particle trajectory and momentum, $50 \leq p \leq 100 \mathrm{MeV} / \mathrm{c}$ for the three most forward pad-rings $\left(17^{\circ}\right.$ to $\left.26^{\circ}\right)$. The observed $\beta$ distribution is peaked at $\sim 1$ with a $\sigma$ of the Gaussian fit of $7.7 \%$.

$\sigma\left(t_{\mathrm{TPC}}\right)$ represents the uncertainty introduced by the TPC track reconstruction. Strictly speaking, this does not represent an uncertainty in the measurement of time in the TPC, but in the prediction of the $\mathrm{ToF}$ of the particle based on track-length and momentum measurements in the TPC. It should be noted that the value of $141 \mathrm{ps}$ for the intrinsic time resolution of the RPCs is measured using the time difference of overlapping chambers and does not include common variations such as the jitter of the TDC start signal and residual common temperature variations.

An estimation of system $t_{\text {tof }}$ uncertainty can be obtained from distributions used to calculate $t 0$ (see Fig. 11). We recall that $t 0$ is a difference between measured time-of-flight and calculated one, based on measured track momentum and length. The sigmas of those fitted distributions are the measure of ToF resolution. The overall time resolution for the different pad-rings measured using pions is given in Fig. 14. The average $\sigma\left(t_{\text {tof }}\right)$ is 305 ps.

Using data from Fig. 14 and (3) one can conclude that the contribution of the TPC track reconstruction to the overall PID capability corresponds to a time resolution of the order of 260 ps, on average. However, this is an overestimate since it is obtained neglecting contributions not included in the 141 ps mentioned above.

Another way of estimating the performance of the system, though in a limited momentum range, is by selecting electrons and positrons, which all have $\beta \approx 1$ here. Fig. 15 represents an $\mathrm{e}^{ \pm}$enriched sample by requiring pairs of "symmetric" tracks with opposite charge, close in "transverse momentum-production angle" space and with common origin. The $\beta$ distribution of these tracks is well centered around one and the $\sigma$ is $9.0 \%$. The average track length and ToF of these tracks are $593 \mathrm{~mm}$ and $1.99 \mathrm{~ns}$, respectively. Expressing $\delta \beta / \beta$ as a time resolution one 


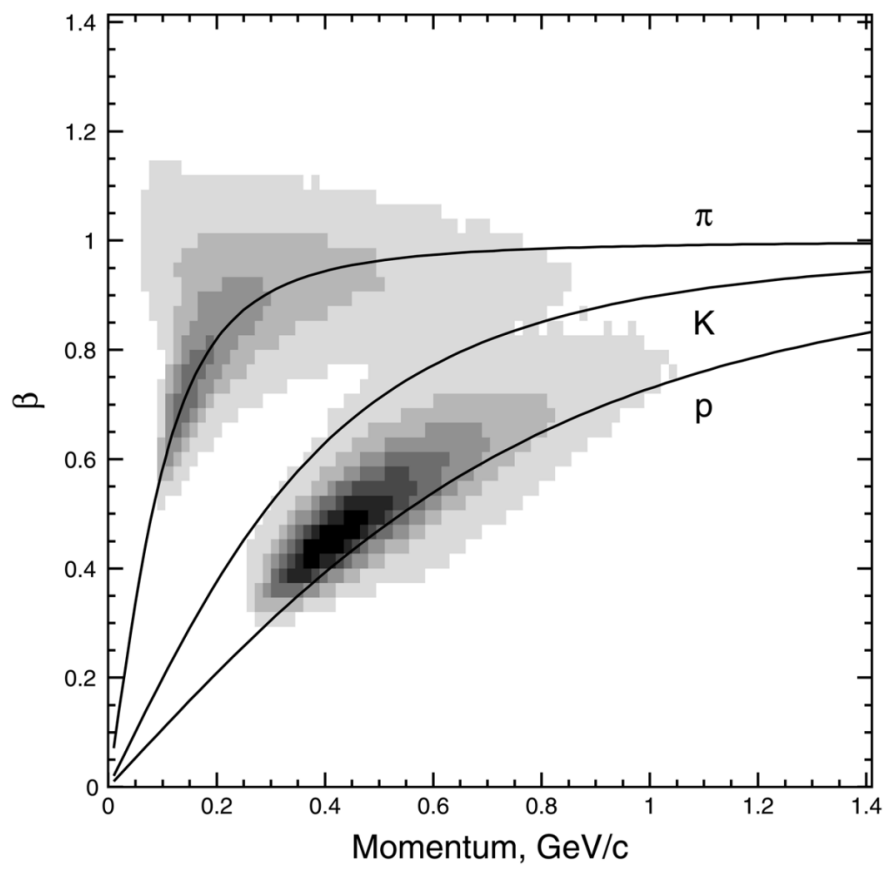

Fig. 17. Relativistic velocity $\beta$, measured by the barrel ToF system, as a function of momentum, measured by the TPC, for all particles. The pion time-charge correction is applied to all signals. The curves represent theoretical dependence for pions (upper curve), kaons (middle curve) and protons (bottom curve). Proton signals are shifted towards higher $\beta$, see discussion in III.C.

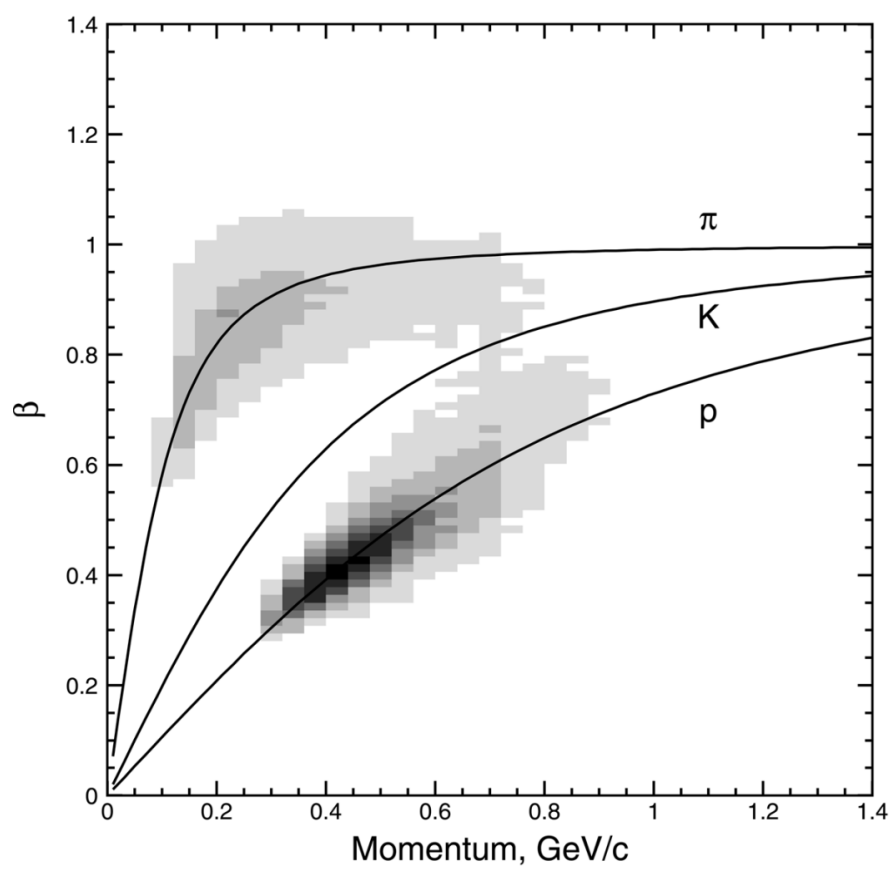

Fig. 18. Relativistic velocity $\beta$, measured by barrel ToF system, as a function of momentum, measured by the TPC, for all particles. Separate time-charge corrections are applied for pions and protons. Now protons are centered on the theoretical (bottom) curve.

obtains a ToF resolution of $\sim 180$ ps. This is much better than the resolution of $305 \mathrm{ps}$ stated above. The apparent discrepancy can be attributed to the fact that electrons in this momentum range $(50 \leq p \leq 300 \mathrm{MeV} / \mathrm{c})$ are fully relativistic and that therefore their predicted $\beta$ is close to unity independently of the (a)

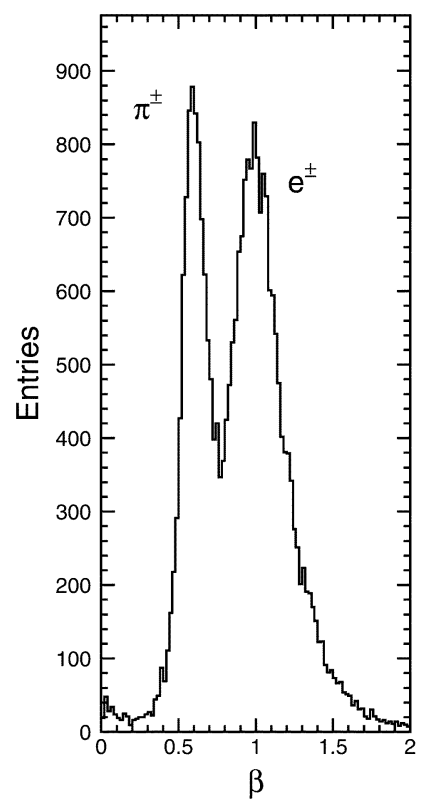

(c)

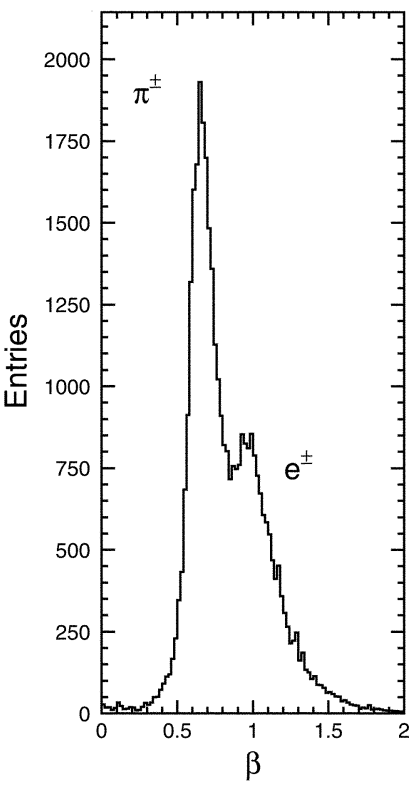

(b)

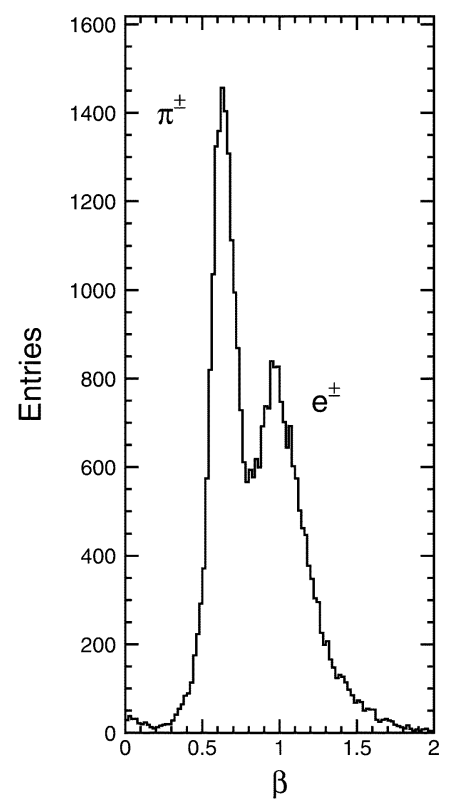

(d)

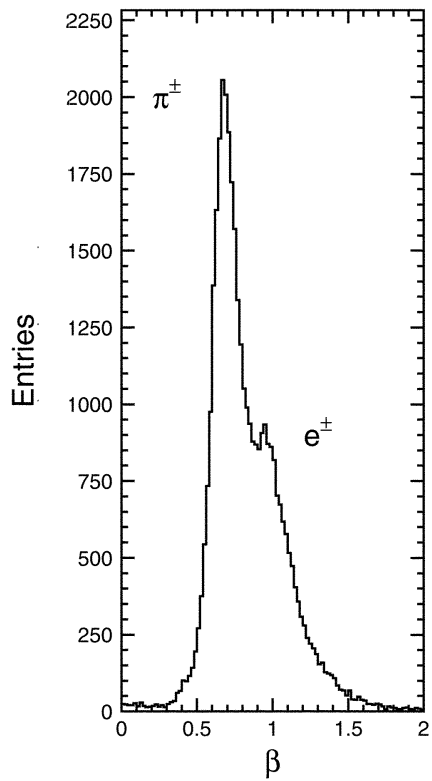

Fig. 19. Relativistic velocity $\beta$ in different momentum slices for all particles. (a) the e peak is centered at $\beta=1$; the $\pi$ peak appears at $\beta \sim 0.6$; (b) the fraction of $\pi$ increases and that of e decreases; (c) $\pi$ peak becomes dominant; (d) the number of electrons is already so small that it is difficult to see them on top of $\pi$ signals. (a) $90<\mathrm{P}_{\text {tot }}<100 \mathrm{MeV} / \mathrm{c}$, (b) $100<\mathrm{P}_{\text {tot }}<110 \mathrm{MeV} / \mathrm{c}$, (c) $110<\mathrm{P}_{\text {tot }}<120 \mathrm{MeV} / \mathrm{c}$, (d) $120<\mathrm{P}_{\text {tot }}<130 \mathrm{MeV} / \mathrm{c}$.

measurement errors in their momentum. In this particular case, the calculated time resolution of $180 \mathrm{ps}$ includes the intrinsic RPC time resolution, beam time resolution and the TPC uncertainties coming from track length measurements. In this estimation the good RPC intrinsic time resolution is demonstrated again in an independent way and the conclusion can be drawn that particle momentum measurements are an important source of ToF uncertainties. From the difference in the two estimates of the overall time resolution, one from electrons with $\beta=1$ (180 ps) and the one from pions with lower $\beta$, one can conclude that the contribution of the momentum resolution to the overall 
(a)

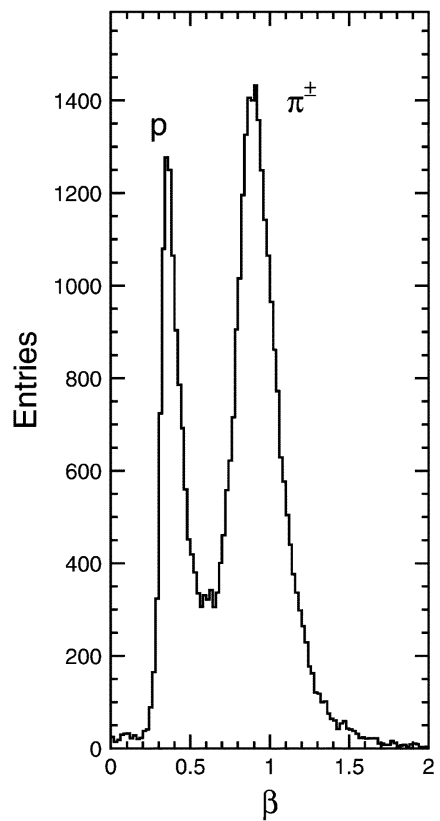

(c)

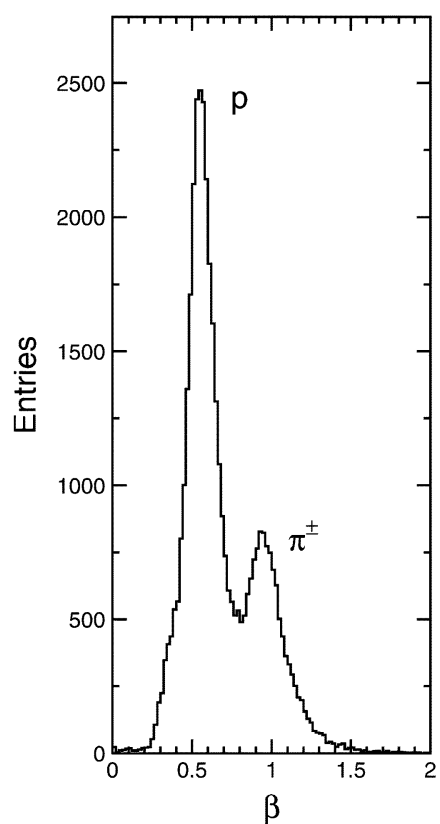

Fig. 20. Relativistic velocity $\beta$ in different momentum slices for all particles. (a) the $\pi$ peak is dominant, centered at $\beta \sim 0.9$; protons appear at $\beta \sim 0.35$; (b) the proton peak rises and becomes dominant; (c) the proton peak is moving to larger $\beta$; (d) number of $\pi$ decreases and their signal is being obscured by the proton tail. (a) $280<\mathrm{P}_{\text {tot }}<290 \mathrm{MeV} / \mathrm{c}$, (b) $400<\mathrm{P}_{\text {tot }}<410 \mathrm{MeV} / \mathrm{c}$, (c) $550<\mathrm{P}_{\text {tot }}<560 \mathrm{MeV} / \mathrm{c}$, (d) $690<\mathrm{P}_{\text {tot }}<700 \mathrm{MeV} / \mathrm{c}$.

PID performance using ToF is similar to that from the time-measurement itself. This is expected since the prediction of $\beta$ for the population of pions used in the analysis depends significantly on the measured momentum. The relative momentum resolution is $\approx 10 \%$ which introduces an uncertainty in $\beta$ similar to the relative time resolution in the region of interest. Again, this is an overestimate of the effect of the momentum resolution, since the time resolution of the pions was measured for a variety of momenta and incident angles, thereby introducing systematic dependences discussed in Section III.C. (a)

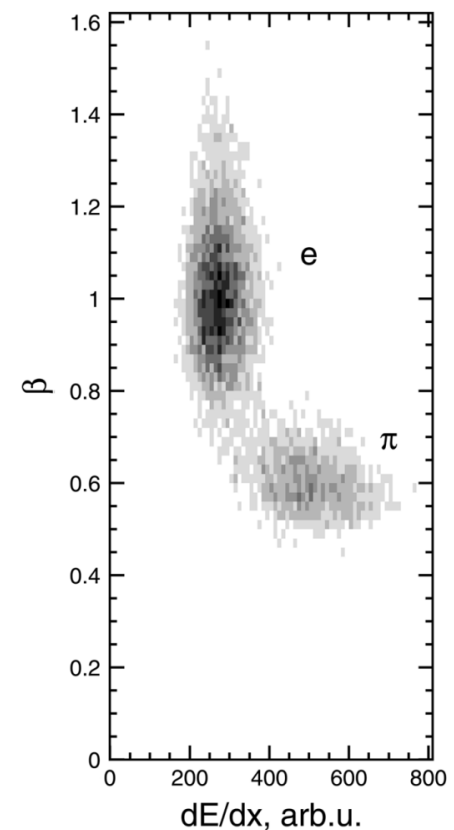

(c)

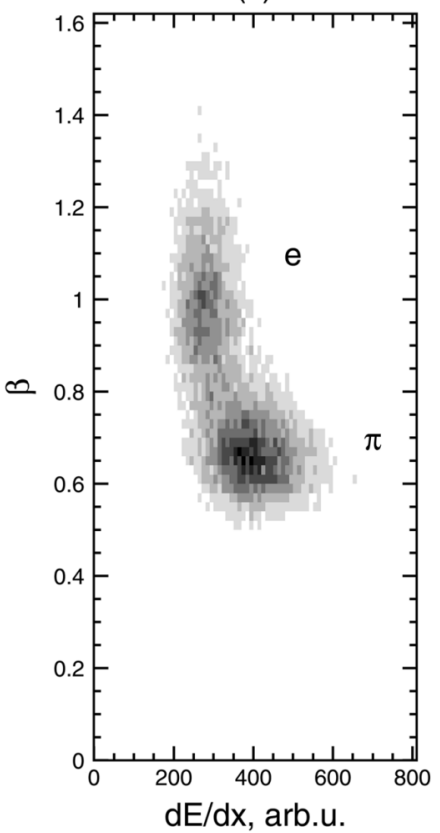

(b)

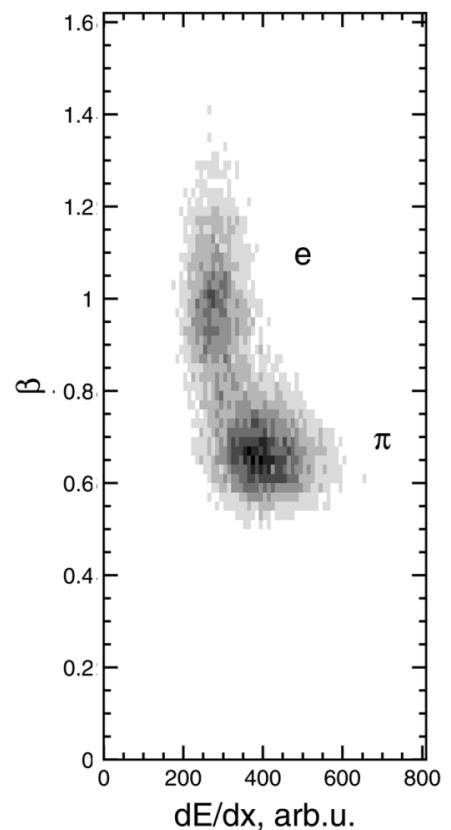

(d)

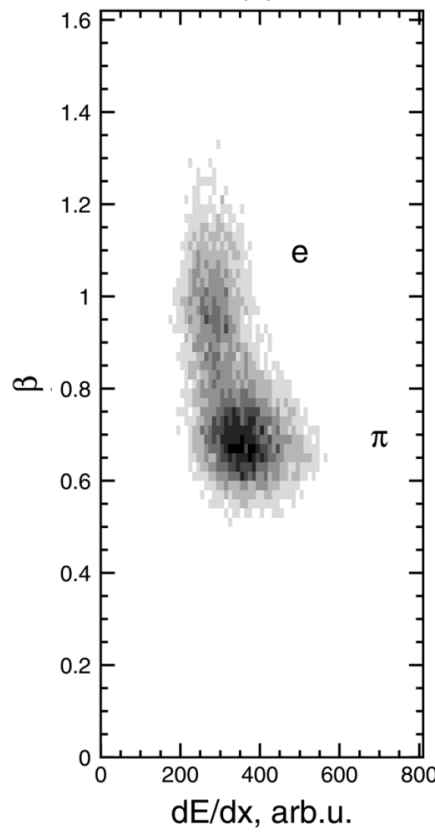

Fig. 21. Relativistic velocity $\beta$ versus $d E / d x$ in different momentum slices for e and $\pi$. (a) the $\mathrm{e}^{ \pm}$area is centered at $\beta=1 ; \pi^{ \pm}$area appears at $\beta \sim 0.6$; (b) the fraction of $\pi^{ \pm}$increases and that of $\mathrm{e}^{ \pm}$decreases; (c) the $\pi^{ \pm}$area becomes dominant; (d) the number of $\mathrm{e}^{ \pm}$is already so small that it is difficult to see them on top of pions with $\beta \sim 1$. (a) $90<\mathrm{P}_{\text {tot }}<100 \mathrm{MeV} / \mathrm{c}$, (b) $100<\mathrm{P}_{\text {tot }}<$ $110 \mathrm{MeV} / \mathrm{c}$, (c) $110<\mathrm{P}_{\text {tot }}<120 \mathrm{MeV} / \mathrm{c}$, (d) $120<\mathrm{P}_{\text {tot }}<130 \mathrm{MeV} / \mathrm{c}$.

For the measurement of pion production at large angles with respect to the beam a significant background contribution of electrons and positrons from $\pi^{0}$ decays is expected at momenta below $300 \mathrm{MeV} / \mathrm{c}$, with higher momenta for smaller production angles. A demonstration of the resolution of the measurement of $\beta$ for the three most forward RPC pad-rings is given in Fig. 16. Electrons and positrons are selected by a constraint on $d E / d x$ in a momentum range between 50 and $100 \mathrm{MeV} / \mathrm{c}$. The observed $\beta$ distribution is peaked at $\sim 1$ with $\sigma$ of $7.7 \%$. 
(a)

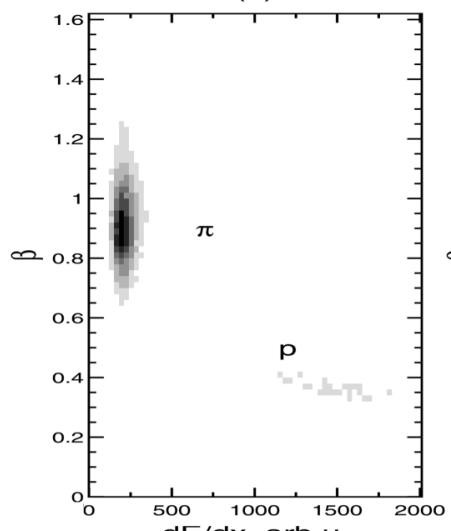

(c)

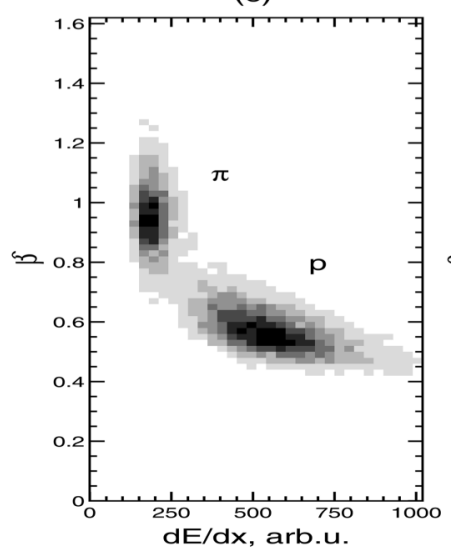

(b)

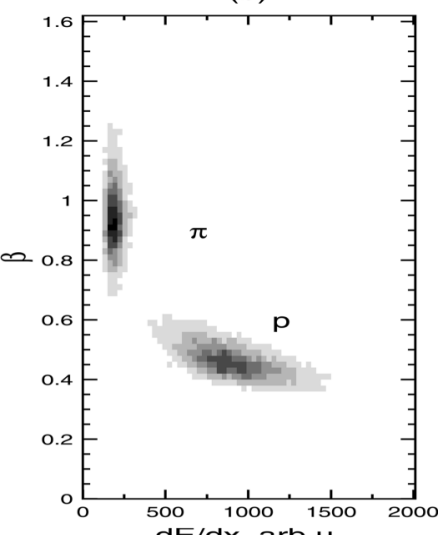

(d)

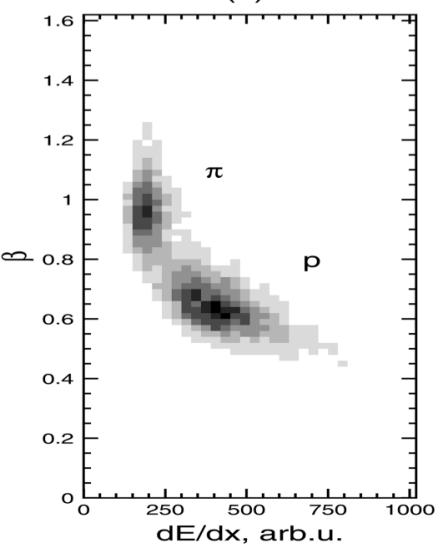

Fig. 22. Relativistic velocity $\beta$ versus $d E / d x$ in different momentum slices for $\pi$ and protons. (a) the $\pi^{ \pm}$area is dominant, centered at $\beta \sim 0.9$; protons appear at $\beta \sim 0.35$; (b) the proton area increases; (c) and (d): the proton area is moving to larger $\beta$ but the separation of both fractions is still clear. (a) $280<$ $\mathrm{P}_{\text {tot }}<290 \mathrm{MeV} / \mathrm{c}$, (b) $400<\mathrm{P}_{\text {tot }}<410 \mathrm{MeV} / \mathrm{c}$, (c) $550<\mathrm{P}_{\text {tot }}<560$ $\mathrm{MeV} / \mathrm{c}$, (d) $690<\mathrm{P}_{\text {tot }}<700 \mathrm{MeV} / \mathrm{c}$.

\section{Particle Identification}

The particle identification capabilities of the barrel ToF system have been evaluated after performing the calibration procedure described above. Fig. 17 demonstrates the correlation between measured relativistic velocity $\beta$ of all particles and their momentum when the pion time-charge correction is used. Proton signals are shifted with respect to the theoretical curve due to difference in their time-charge dependence, as discussed in Section III.C. If one applies separate time-charge corrections for pions and protons, the signals become well centered around the theoretical curves, as is seen in Fig. 18. The e/ $\pi$ separation capabilities of the system are better seen in Fig. 19, where distributions of measured $\beta$ are shown for different momentum slices. Similar plots are presented in Fig. 20 for proton/pion separation. Another even more promising approach is a presentation of particle velocity $\beta$ versus energy losses $d E / d x$. Well defined areas populated by signals of particles of a given type can easily be separated for different momentum ranges as illustrated in Figs. 21 and 22.

\section{Efficiency}

The RPC pad efficiency is defined as a ratio of a number of charged tracks producing a hit in a given pad to the total number of TPC tracks crossing it. This ratio gives a lower bound of (a)

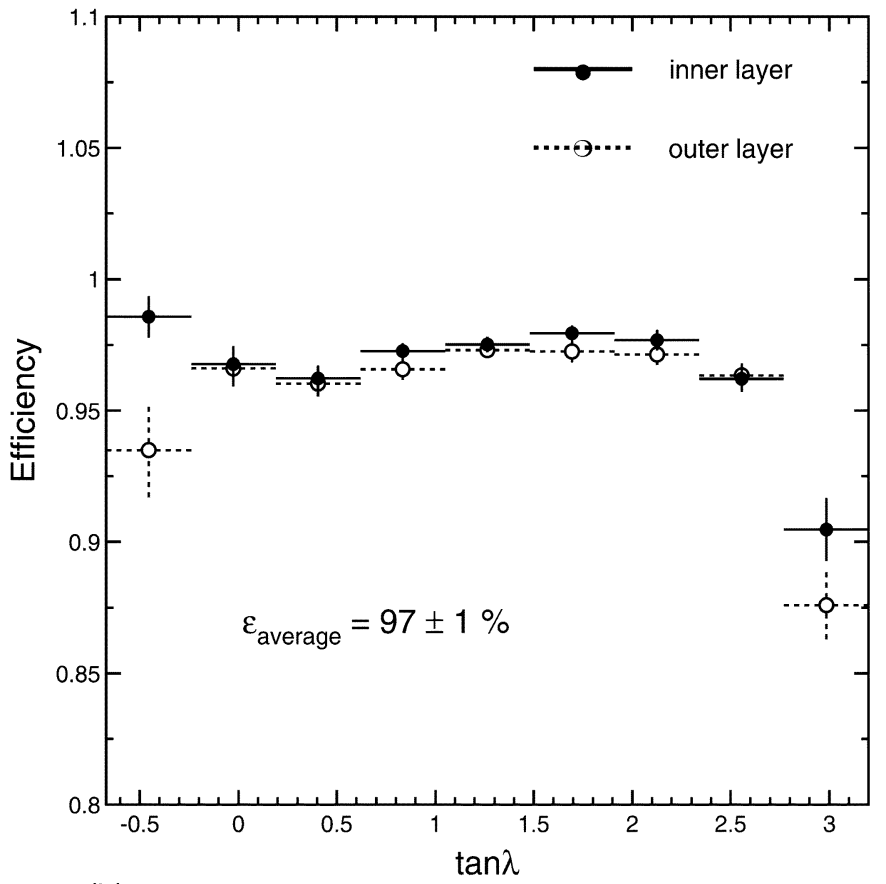

(b)

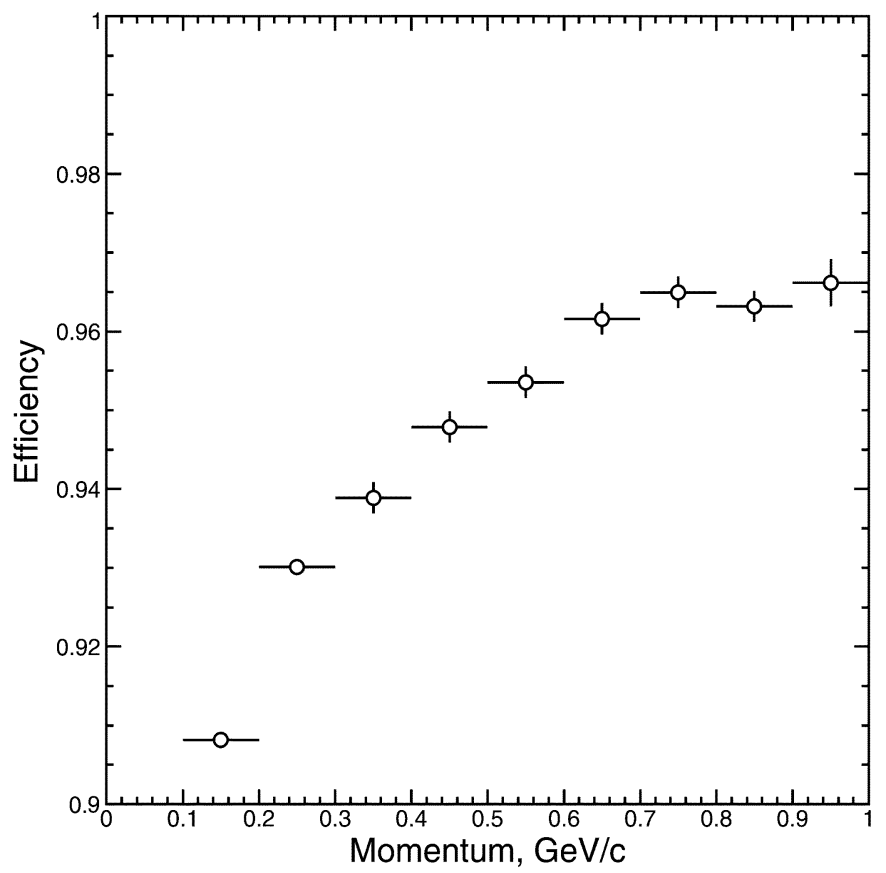

Fig. 23. (a) RPC efficiency for pions as function of $\tan \lambda$ for the inner and outer layer for $p>0.7 \mathrm{GeV} / \mathrm{c}$ and (b) RPC efficiency for pions as function of total momentum.

the RPC efficiency as the purity of TPC reconstruction is not $100 \%$ and also the algorithm which extrapolates TPC tracks to the RPC has finite precision. Moreover, some of the low-momentum heavily ionizing particles reconstructed in the TPC do not reach the RPC chambers because they stop in the material between the TPC and the RPC. In order to minimize possible bias only very well reconstructed tracks in the TPC are selected.

The top plot in Fig. 23 shows the efficiency for pions as a function of $\tan \lambda$ for inner and outer layers, where $\lambda$ is the angle 
between the track and the plane perpendicular to the beam direction. The highest efficiency is observed in the central part of the barrel. The outer layer has a lower apparent efficiency because the particles traverse more material compared to the inner one. The bottom plot in Fig. 23 shows the efficiency for pions as a function of particle momentum. It is seen that the measured efficiency increases with momentum.

\section{CONCLUSION}

The HARP RPC barrel, built of 30 thin four gap chambers, has been investigated and calibrated using reconstructed tracks produced by beams with a number of beam energies impinging on different targets. Various effects such as time-charge and temperature dependencies have been studied, understood, parametrized and corrections for these have been implemented. The intrinsic RPC time resolution of the chambers of 141 ps and a ToF resolution of the full system of $180 \mathrm{ps}$ have been obtained. RPC particle identification capabilities are demonstrated. The effective resolution of the comparison of predicted and measured $\mathrm{ToF}$ is $305 \mathrm{ps}$ in the region of interest for this experiment. The efficiency for pions is determined to be about $97 \%$, on average.

\section{ACKNOWLEDGMENT}

The authors would like to thank S. Borghi for the fruitful discussions on reconstruction matters in the TPC, C. Wiebusch for the measurement of the TDC channel widths, and their HARP colleagues for the deep and thorough discussions during HARP analysis meetings.

\section{REFERENCES}

[1] M. G. Catanesi, "Proposal to study hadron production for the neutrino factory and for the atmospheric neutrino flux," Dec. 1999, CERNSPSC/99-35.

[2] M. G. Catanesi, "The HARP detector at the CERN PS," Nucl. Instrum. Methods Phys. Res. A, to be published.

[3] M. Bogomilov, "The HARP RPC time-of-flight system," Nucl. Instrum. Methods Phys. Res. A, vol. A508, pp. 152-158, Aug. 2003.

[4] A. Akindinov, "A four-gap glass-rpc time-of-flight array with 90 ps time resolution,” IEEE Trans. Nucl. Sci., vol. 48, no. 5, pp. 1658-1663, Oct. 2001.

[5] M. G. Catanesi, HARP Collaboration, "Measurement of the production cross-section of positive pions in p Al collisions at $12.9-\mathrm{GeV} / \mathrm{c}$," $\mathrm{Nucl}$. Instrum. Methods Phys. Res. B, vol. B732, pp. 1-45, Jan. 2006.

[6] G. Barr, "Performance of multigap RPC detectors in the HARP experiment," Nucl. Instrum. Methods Phys. Res. A, vol. A533, pp. 214-220, Nov. 2004

[7] M. Bogomilov, "Resistive Plate Chambers in the HARP Experiment," Ph.D. dissertation, Faculty of Physics, Dept. Atomic Physics, St. Kliment Ohridski Univ., Sofia, Bulgaria, May 2007. 